\title{
Neural Correlates of Urinary Retention in Lateral Medullary Infarction
}

\author{
Appaswamy Thirumal Prabhakar, Atif Shaikh Iqbal Ahmed, Aditya Vijayakrishnan Nair, Vivek Mathew, Sanjith Aaron, \\ Ajith Sivadasan, Mathew Alexander \\ Department of Neurological Sciences, Christian Medical College, Vellore, India
}

Purpose: The brainstem plays an important role in the control of micturition, and brainstem strokes are known to present with micturition dysfunction. Micturition dysfunction in cases of lateral medullary infarction (LMI) is uncommon, but often manifests as urinary retention. In this study, we investigated the neuro-anatomical correlates of urinary retention in patients with LMI.

Methods: This was a hospital-based retrospective study conducted in the neurology unit of a quaternary-level teaching hospital. Inpatient records from January 2008 to May 2018 were searched using a computerized database. Cases of isolated LMI were identified and those with micturition dysfunction were reviewed. MRI brain images of all patients were viewed, and individual lesions were mapped onto the Montreal Neurological Institute (MNI) space manually using MRIcron. Nonparametric mapping toolbox software was used for voxel-based lesion-symptom analysis. The Liebermeister test was used for statistical analysis, and the resultant statistical map was displayed on the MNI template using MRIcron.

Results: During the study period, 31 patients with isolated LMI were identified. Their mean age was 48 years and 28 (90\%) were male. Six of these patients (19\%) developed micturition dysfunction. All 6 patients had urinary retention and 1 patient each had urge incontinence and overflow incontinence. In patients with LMI, the lateral tegmentum of the medulla showed a significant association with urinary retention.

Conclusions: In patients with isolated LMI, we postulate that disruption of the descending pathway from the pontine micturition centre to the sacral spinal cord at the level of the lateral tegmentum results in urinary retention.

Keywords: Lateral medullary infarction; Urinary retention; Neural control

- Research Ethics: The study was approved by the Institutional Review Board and ethics committee of Christian Medical College, Vellore (IRB

No. 11542). Informed consent was not obtained, as this was a retrospective hospital record-based study.

- Conflict of Interest: No potential conflict of interest relevant to this article was reported.

\section{- HIGHLIGHTS}

- Micturition dysfunction can be seen in patients with lateral medullary infarction.

- Lesions involving the lateral tegmentum of the medulla can result in urinary retention.

\section{INTRODUCTION}

Micturition dysfunction is a common problem following acute stroke, with a prevalence of $37 \%-79 \%$ [1-3]. The brainstem plays an important role in the control of micturition, and brainstem strokes are known to present with micturition dysfunction

Corresponding author: Appaswamy Thirumal Prabhakar

(iD https://orcid.org/0000-0003-2008-1808

Department of Neurological Sciences, Christian Medical College, Vellore

632004, India

E-mail: atprabhakar@gmail.com / Tel: +914162282018 / Fax: +914162232035

Submitted: August 3, 2018 / Accepted after revision: August 1, 2019 
$[3,4]$. At the level of the brainstem, the pontine micturition centre (PMC), which is located in the dorsal pontine tegmentum, coordinates the normal micturition reflex [5]. Stroke patients with lesions above the pontine level are likely to have bladder storage dysfunction, while those with lesions at and below the pontine level are likely to have bladder emptying dysfunction [3]. Micturition dysfunction in cases of medullary infarction is uncommon, but often presents with urinary retention [6-8]. The cause of urinary retention in such patients is detrusor underactivity, which has been previously shown in patients with lateral medullary infarction (LMI) [6]. However, limited human data exists regarding which specific areas in the lateral medulla are involved in the control of micturition [6]. The aim of this study was to identify the neuroanatomical sites associated with urinary retention by studying the imaging data of patients who presented with isolated LMI.

\section{MATERIALS AND METHODS}

This was a hospital-based retrospective study conducted in the neurology unit of a quaternary care hospital in India. The study was approved by the Institutional Review Board and ethics committee of Christian Medical College, Vellore (IRB No. 11542). Informed consent was not obtained, as this was a retrospective hospital record-based study. Inpatient records from January 2008 to May 2018 were searched using a computerized database, and records with a diagnosis of LMI were identified. After reviewing the neuro-imaging, patients with isolated LMI were included in the study. Demographic and clinical data were collected from the included patients. Patients with micturition dysfunction were identified by reviewing their medical records, urology consultation notes, and results of urodynamic studies. Brain magnetic resonance imaging (MRI), including T2weighted, fluid-attenuated inversion recovery (FLAIR), and diffusion-weighted imaging (DWI) sequences were viewed, and the individual lesions were mapped onto the MontrealNeurological Institute (MNI) space manually using MRIcron (https://www.nitrc.org/projects/mricron) [9]. Individual lesions were entered into a voxel-based lesion-symptom mapping (VLSM) analysis using the nonparametric mapping toolbox (NPM) from the MRIcron software package (May 2016) [10]. In order to increase the statistical power and to create an overlay map, all lesions were flipped onto the left side [11]. Lesion overlap was first calculated and a color-coded overlay map of injured voxels across all patients and patients with micturition dysfunction was created. The statistical contribution of the lesion location to micturition dysfunction was tested using VLSM in the NPM software. Patients with urinary retention attributed to a neurological cause after ruling out local urological causes were included in the VLSM analysis. The Liebermeister test was performed for each voxel by taking the presence of urinary retention as the dependent variable and comparing patients with a lesion to those without a lesion [10]. Voxels were required to be affected in at least 5 patients to be included in the test [12]. To correct for multiple comparisons, all result maps were corrected using a threshold of a 5\% false discovery rate (FDR). The resultant statistical map was displayed on the MNI template using MRIcron. The location of these voxels was further categorized into anatomical sites such as the rostral, middle, and caudal parts of the medulla, as described by Kim et al. [13].

\section{RESULTS}

Thirty-one inpatient records from January 2008 to May 2018 were identified to have isolated LMI. Of the 31 patients with LMI, 28 were male, with a mean age of 48 years. According to the review of patients' medical records, 8 patients had documented micturition dysfunction. However, 2 patients were excluded from the study as their urological evaluation was suggestive of a bladder outlet dysfunction secondary to prostatomegaly. The demographic and clinical characteristics of the patients with and without micturition dysfunction are given in Table 1. There was no significant difference between the demographic and clinical profiles of the 2 groups, except for the presence of hiccups, which was significantly higher in the group with urinary retention. Of the 6 patients with urinary retention, all had undergone a urological evaluation, but only 1 patient had a urodynamic study done. None of the included patients had bladder outlet obstruction or urinary tract infection at the time of presentation. All 6 patients presented with acute urinary retention within 5 days from the onset of LMI symptoms. One patient had urinary urge incontinence, and 1 patient presented with overflow incontinence. Detrusor underactivity with normal bladder sensation was documented in the patient who underwent a urodynamic study on day 10 from symptom onset. All 31 patients underwent brain MRI including T2, T1, FLAIR, and DWI sequences within 7 days from symptom onset. In the DWI sequences, 4 patients had right-sided lesions, and 2 showed lesions on the left side. The mean infarct volume was 
Table 1. Subject demographics and clinical characteristics

\begin{tabular}{lcccc}
\hline Characteristic & $\begin{array}{c}\text { Total number of } \\
\text { patients with LMI }\end{array}$ & $\begin{array}{c}\text { LMI without urinary } \\
\text { retention }\end{array}$ & $\begin{array}{c}\text { LMI with urinary } \\
\text { retention }\end{array}$ & P-value \\
\hline No. of subjects & 31 & 25 & 6 & 0.59 \\
Age (yr) & $47.97 \pm 17.43$ & $51.5 \pm 20.9$ & $47.1 \pm 16.9$ & 0.49 \\
Sex, male:female & $28: 3$ & $23: 2$ & $5: 1$ & 0.65 \\
Hypertension & 21 & 17 & 4 & 0.35 \\
Diabetes mellitus & 11 & 8 & 3 & 0.24 \\
Smoking & 14 & 10 & 4 & 0.11 \\
Alcohol use & 7 & 4 & 3 & 0.19 \\
Dysphagia & 30 & 25 & 5 & 1.00 \\
Dysarthria & 31 & 25 & 6 & 0.80 \\
Ataxia & 30 & 24 & 6 & 0.01 \\
Hiccups & 11 & 6 & 5 & 0.28 \\
Horner syndrome & 20 & 15 & 5 & 0.22 \\
Facial weakness & 9 & 6 & 3 & 0.65 \\
Impaired gag reflex & 29 & 23 & 6 & 0.38 \\
Crossed sensory loss & 18 & 14 & 4 & 0.67 \\
Pain & 6 & 5 & 1 & 0.78 \\
Prostate volume $\left(\mathrm{cm}^{3}\right)$ & $32.0 \pm 5.2$ & $31.5 \pm 2.7$ & $32.16 \pm 5.7$ & 0.68 \\
Duration of hospital stay (day) & $13.03 \pm 10.4$ & $14.7 \pm 6.8$ & $12.6 \pm 11.2$ & \\
\hline
\end{tabular}

Values are presented as mean \pm standard deviation or number. LMI, lateral medullary infarction.

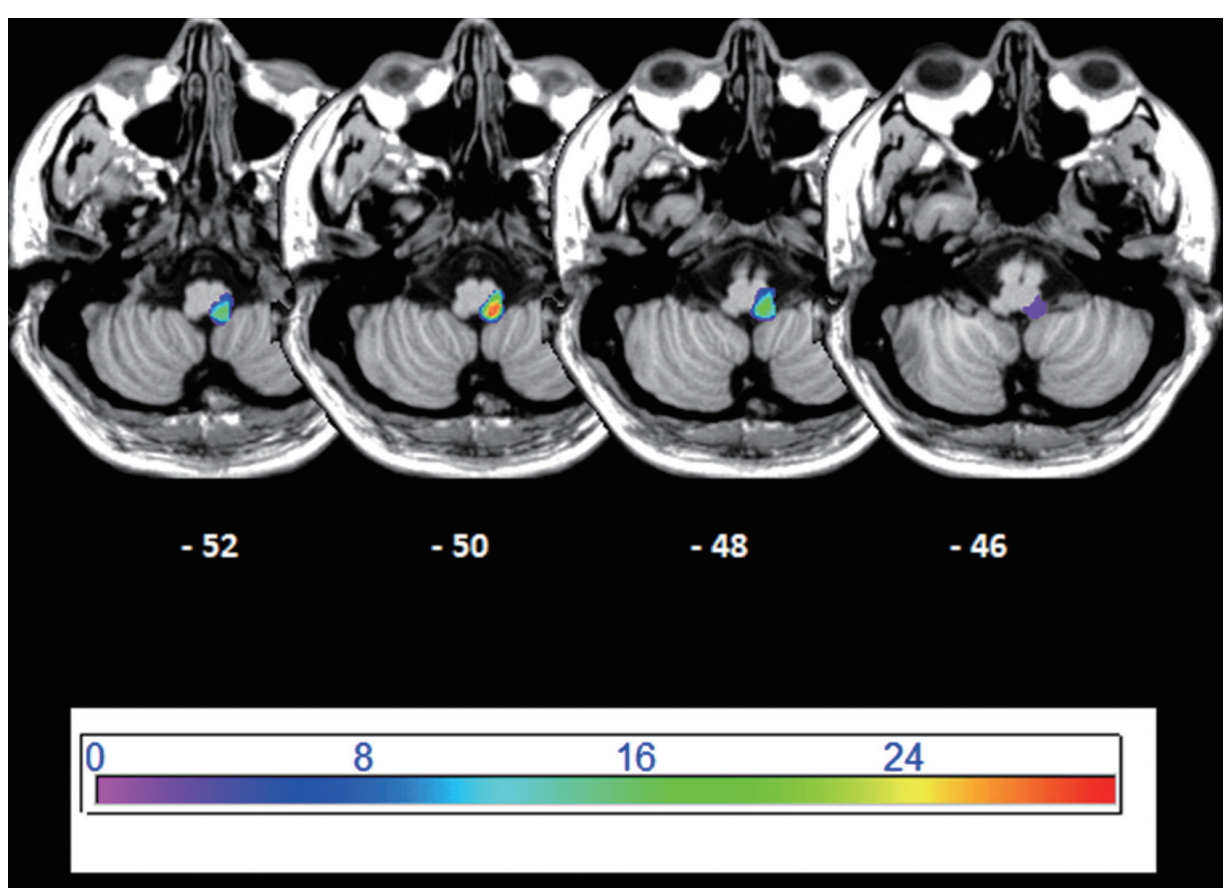

Fig. 1. Lesion overlay map of the overlay map of the individual lesions of all 31 patients with lateral medullary infarction. Maps were overlaid on a T1-template in Montreal Neurological Institute (MNI) space $\left(1 \mathrm{~mm}^{3} \times 1 \mathrm{~mm}^{3} \times 1 \mathrm{~mm}^{3}\right)$. All lesions were flipped to the left side. The MNI coordinates of each axial section ( $z$-axis) is given below each slice. The colour scale indicates the number of patients with a lesion in a particular voxel. 


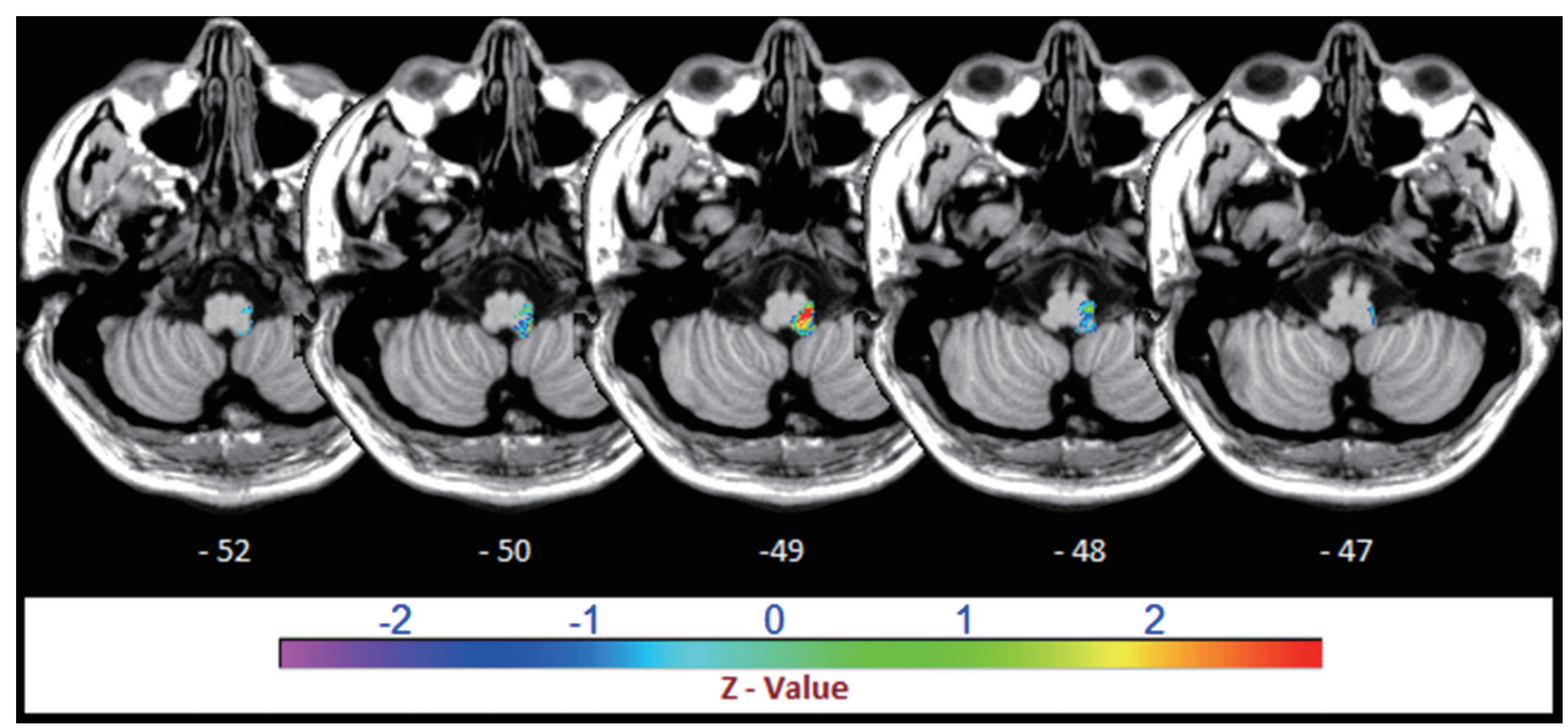

Fig. 2. Statistical map of voxel-based lesion-symptom mapping displaying voxels with a significant association between lesion location and urinary retention. The colour scale indicated the z-statistics from the results of the Liebermeister test. The tests were corrected for multiple comparisons at the level of a 5\% false discovery rate. Statistical maps were overlaid on a T1-template in Montreal Neurological Institute $(\mathrm{MNI})$ space $\left(1 \mathrm{~mm}^{3} \times 1 \mathrm{~mm}^{3} \times 1 \mathrm{~mm}^{3}\right)$. The MNI coordinates of each axial section $(z$-axis) are given below each slice. The areas in red indicate a significant association between a lesion in this voxel and urinary retention. In patients with lateral medullary infarction the lateral tegmentum of the middle medulla was significantly associated with urinary retention.

$1.6 \pm 0.3 \mathrm{~mL}$, and there was no significant difference between the 2 groups. The lesion overlay map of the 31 patients with LMI is given in Fig. 1. In most patients, the LMI lesions were distributed across the lateral medulla. However, 2 patients had lesions isolated to the caudal and rostral medulla. Fig. 2 shows the statistical maps from the VLSM analysis, displaying voxels that showed a significant association between lesion location and urinary retention. The colour scale indicates the z-scores from the Liebermeister test. The statistical maps were overlaid on a T1-template in MNI space $\left(1 \mathrm{~mm}^{3} \times 1 \mathrm{~mm}^{3} \times 1 \mathrm{~mm}^{3}\right)$. The areas mapped in red denote voxels with a statistically significant association with urinary retention at an FDR level of 5\%. From the location of these voxels, the corresponding anatomical site in the medulla was identified. In patients with LMI, the lateral tegmentum of the medulla showed a significant association with urinary retention.

\section{DISCUSSION}

Normal bladder function consists of a storage phase and a micturition phase, which are controlled by a hierarchical system of neural networks that are integrated at the level of the forebrain, brainstem, and spinal cord. A spino-bulbo-spinal reflex with a complex switching mechanism is involved in the initiation of micturition. During the storage phase, afferent signals from the bladder ascend through the spinal cord to synapse in the midbrain periaqueductal grey (PAG), which in turn, may have an inhibitory effect on the pons. When the afferents exceed a certain threshold, secondary to bladder filling, activation of the PMC occurs. The PMC receives excitatory input from bladder afferents indirectly via the ventrolateral PAG and sends descending glutamatergic projections to the sacral spinal cord via the lateral funiculus [14]. The descending efferents result in detrusor contraction via the excitatory parasympathetic pathway and sphincter relaxation through the nucleus of Onuf [15]. A pontine region located ventrolateral to the PMC (the L-region) sends a direct excitatory projection to the nucleus of Onuf, promoting contraction of the pelvic floor and sphincter, thereby maintaining continence [14]. Lesions affecting these descending fibers can disrupt the excitatory input to the bladder, resulting in detrusor underactivity and urinary retention. Urinary retention has been previously described in LMI, and is the com- 
monest micturition dysfunction [1,2,6,7]. Based on studies in cats, 2 descending pathways between the PMC and the spinal cord have been noted: a direct pathway and an indirect pathway, with connections to the medullary neurons [16]. Neurons in the nucleus reticularis magnocellularis of the medulla were found to receive input from the PMC, fire during micturition, and project to the spinal cord [16]. Additionally, the neurons in PMC have projections through the lateral tegmentum of the medulla, specifically between the inferior olive and lateral reticular nucleus, to the sacral cord [17]. Electrical stimulation of this region is known to produce an increase in bladder pressure [5]. In a lesion analysis study in humans with LMI by Cho et al. [6], the lateral tegmentum was identified as the site associated with urinary retention. This conclusion was drawn by subtraction of the overlapped lesion maps in the control group from those in the patient group. However, a statistical analysis using VLSM was not done. In our study, 19.4\% of the patients with isolated LMI had urinary retention. This was similar to the results of Cho et al. [6], who found a prevalence of urinary retention of $16 \%$. Using a VSLM analysis, we identified the lateral tegmentum of the medulla as the neuro-anatomical area associated with micturition. Our results corroborate the findings of Cho et al, and adds evidence supporting the postulate that the descending pathway from the PMC in humans may be identical to the pathway in cats $[6,17]$. Although the PMC projects bilaterally to the sacral cord, right-sided lesions are more likely to produce urinary retention [6]. Additionally, positron-emission tomography studies in humans during micturition show a right-predominant pattern of pontine activation [18]. In our study, we likewise found that 4 of the 6 patients with urinary retention had right-sided lesions. We also found an association between intractable hiccups and urinary retention. Park et al. [19] in a lesion-based study of patients with LMI presenting with hiccups, found that middle-level and dorsolateral lesion locations were associated with hiccups. This is the same region that we identified in our study to be associated with urinary retention $[19,20]$. The main limitations of our study include the retrospective study design, the nonavailability of urodynamic studies on all patients, and the availability of limited data on the recovery of bladder symptoms during follow-up. The major strength of our study is that it is a lesion-symptom mapping study powered with statistical testing, unlike previous lesionbased studies on LMI and urinary retention [6].

In conclusion, $19 \%$ of the patients who presented with isolated LMI had urinary retention. We postulate that disruption of the descending pathway from the PMC to the sacral spinal cord, at the level of the lateral tegmentum of the medulla, is the likely cause of urinary retention in patients with LMI.

\section{AUTHOR CONTRIBUTION STATEMENT}

- Full access to all the data in the study and takes responsibility for the integrity of the data and the accuracy of the data analysis: ATP

-Study concept and design: ATP, ASIA, AVN

- Acquisition of data: ATP

- Analysis and interpretation of data: ATP

- Drafting of the manuscript: ATP

- Critical revision of the manuscript for important intellectual content: ATP, ASIA, VM, SA, AS, MA

- Statistical analysis: ATP, ASIA

- Obtained funding: ATP

- Administrative, technical, or material support: ATP, VM, MA

- Study supervision: ATP, VM

\section{REFERENCES}

1. Burney TL, Senapati M, Desai S, Choudhary ST, Badlani GH. Acute cerebrovascular accident and lower urinary tract dysfunction: a prospective correlation of the site of brain injury with urodynamic findings. J Urol 1996;156:1748-50.

2. Umemura $T$, Ohta $H$, Yokota $A$, Yarimizu S, Nishizawa S. Urinary retention associated with stroke. J UOEH 2016;38:263-9.

3. Yum KS, Na SJ, Lee KY, Kim J, Oh SH, Kim YD, et al. Pattern of voiding dysfunction after acute brainstem infarction. Eur Neurol 2013;70:291-6.

4. Lee HS, Choi JG, Shin JH. Urological disturbance and its neuroanatomical correlate in patients with chronic brainstem stroke. Neurourol Urodyn 2017;36:136-41.

5. Loewy AD, Saper CB, Baker RP. Descending projections from the pontine micturition center. Brain Res 1979;172:533-8.

6. Cho HJ, Kang TH, Chang JH, Choi YR, Park MG, Choi KD, et al. Neuroanatomical correlation of urinary retention in lateral medullary infarction. Ann Neurol 2015;77:726-33.

7. Lee KB, Jang IM, Roh H, Ahn MY, Woo HY. Transient urinary retention in acute right lateral medullary infarction. Neurologist 2008;14:312-5.

8. Sung SM, Shin JY, Cho KY, Kang TH, Cho HJ. Bladder storage disorder due to detrusor overactivity in a patient with acute medial medullary infarction. J Neurol Sci 2015;357:307-9. 
9. Rorden C, Brett M. Stereotaxic display of brain lesions. Behav Neurol 2000;12:191-200.

10. Rorden C, Karnath HO, Bonilha L. Improving lesion-symptom mapping. J Cogn Neurosci 2007;19:1081-8.

11. Cheng B, Forkert ND, Zavaglia M, Hilgetag CC, Golsari A, Siemonsen $S$, et al. Influence of stroke infarct location on functional outcome measured by the modified rankin scale. Stroke 2014; 45:1695-702.

12. Medina J, Kimberg DY, Chatterjee A, Coslett HB. Inappropriate usage of the Brunner-Munzel test in recent voxel-based lesionsymptom mapping studies. Neuropsychologia 2010;48:341-3.

13. Kim K, Lee HS, Jung YH, Kim YD, Nam HS, Nam CM, et al. Mechanism of medullary infarction based on arterial territory involvement. J Clin Neurol 2012;8:116-22.

14. Benarroch EE. Neural control of the bladder: recent advances and neurologic implications. Neurology 2010;75:1839-46.

15. Kitta T, Mitsui T, Kanno Y, Chiba H, Moriya K, Shinohara N.
Brain-bladder control network: the unsolved 21st century urological mystery. Int J Urol 2015;22:342-8.

16. Sugaya K, Ogawa Y, Hatano T, Nishijima S, Matsuyama K, Mori S. Ascending and descending brainstem neuronal activity during cystometry in decerebrate cats. Neurourol Urodyn 2003;22:343-50.

17. Holstege G, Griffiths D, de Wall H, Dalm E. Anatomical and physiological observations on supraspinal control of bladder and urethral sphincter muscles in the cat. J Comp Neurol 1986;250:449-61.

18. Nour S, Svarer C, Kristensen JK, Paulson OB, Law I. Cerebral activation during micturition in normal men. Brain 2000;123(Pt 4):781-9.

19. Park MH, Kim BJ, Koh SB, Park MK, Park KW, Lee DH. Lesional location of lateral medullary infarction presenting hiccups (singultus). J Neurol Neurosurg Psychiatry 2005;76:95-8.

20. Chang YY, Chen WH, Liu JS, Shih PY, Chen SS. Intractable hiccup caused by medulla oblongata lesions. J Formos Med Assoc 1993; 92:926-8. 